\title{
Stimulus correlates of visual pattern discrimination and the problem of grain
}

\author{
LEONARD ZUSHE \\ UNIVERSITY OF TULSA
}

A recent paper by V. J. Polidora (1966) is criticized on the basis that the experimental design did not provide an adequate test of the usefulness of 15 visual form parameters, mainly because of differences in the grain of the matrices used to construct and measure stimuli.

In a paper published recently in this journal, Polidora (1966) reports the use of 15 parameters of metric visual patterns as predictors of discrimination performance by monkeys. Of the 15 measures used, nine were especially defined by polidora for use with a type of metric pattern, while six others had been used by other investigators with polygons. Three of these had been originated by Zusne (1965) and three by Attneave and Arnoult (1956) and Hochberg, Gleitman, and McBride (1948). In a preliminary analysis of variance in which the effects of four of Polidora's measures were analyzed, each measure yielded a significant main effect, a finding reported in a number of Polidora's earlier experiments with monkeys. The data were then treated by two techniques of multidimensional analysis. Both techniques showed up only one of the 15 parameters, Unique Elements (UE), as a statistically significant, independent contributor to response variance. This last result must be questioned, however, for the following reasons: (a) only Polidora's measures were varied systematically, the variation of the rest of the independent variables was left to chance, but the statistical analysis considered the contributions of all; (b) all measures were pitted against one, Unique Elements, which, by definition, reflected any and all possible differences between two patterns, hence correlated with every one of the measures used; (c) the potential contribution of measures that were not Polidora's were minimized by computing them in the same very coarse-grained matrix within which the metric stimuli had been constructed but which renders any other measure meaningless.

First, the procedure of varying systematically four of the 15 variables and leaving the variation of the rest to chance is questionable. Unless these other variables are correlated with these four, they will vary randomly, and it will be impossible to determine their respective contributions. As it turned out, both the discriminant analysis technique and partial correlation coefficients showed only the UE measure to be a significant predictor of response. The validity vector obtained by the multiple regression technique, however, showed two of Polidora's four measures and four of their derivations significantly favored over the others.
In the second place, none of the measures used were given a fair test due to the indeed unique nature of the UE measure. This measure is obtained on two metric patterns, constructed in a square matrix, by comparing each pair of corresponding cells in the two matrices on whether they are lighted or not and by counting the number of pairs in which the two cells are in different states. On this basis, any kind of a difference will be reflected as some value of UE: differences in complexity, area, rotation, and contour. Even two identical shapes will produce a non-zero UE measure if one is translated with regard to the other. Thus the UE measure is a measure not only of differences between two figures but also of differences between a figure and the ground occupied by the matrix within which the second figure is contained. Such versatility may ve a virtue where it is unnecessary to know which stimulus property was actually utilized by an organism in discriminating between two shapes. However, when some organisms fail to respond to differences that are clearly discriminable by others, the UE interpretation fails. Precisely such findings have been reported by the same authors (e.g., Sutherland) whose experimental results Polidora attempts to reinterpret on the basis of UE. In the present case the UE measure played a different role. Because of its versatility it must correlate with any other imaginable measure of difference between two patterns, including the other 14 measures used by Polidora. As a result and by virtue of the statistical treatment given the data it simply had to be the only measure to correlate with the subjects' response significantly.

The third reason is the problem of matrix grain. In Polidora's experiment both the various moments of area and Attneave's measures based on the perimeter and area failed to make independent contributions to response variance. The reason for this has already been indicated. The second moment of area and Attneave's "D," however, did show significant correlations with response before the effect of intercorrelation was eliminated, but they were quite low. There are two possible reasons for this. One concerns only the moments of area. On page 412 of Polidora's paper there is the statement that when one of a pair of identical patterns is rotated or displaced, none of the 15 measures used would yield a difference measure save for the UE measure. This is true only for the case of translation, but then one does not expect these measures to reflect this operation since they are measures of figure, not of ground. In the case of 
rotation, however, all uneven (3rd, 5th, etc.) moments will change at least their algebraic signs if the rotation is one of 180 degrees, and the value of all moments will change for any other amount of rotation as well as reflection. If Polidora's patterns did not show differences in the values of moments with rotations it means that these measures were either not properiy computed or used.

The second reason for the poor showing of measures that were not Polidora's lies in the extremely coarse grain of the matrix used to construct the stimuli. While Polidora's measures work fine with patterns constructed in a 4 by 4,3 by 3 , or even a 2 by 2 matrix, such a coarse grain renders any other measure on these patterns useless. The second moment of area, for example, is a measure of variance. The largest number of lighted cells in Polidora's 4 by 4 matrix was 11 , but how meaningful is the variance computed with an $\mathrm{N}$ of 11 ? Just adding or subtracting one element changes the value of a moment drastically, especially the values of the higher moments. The same goes for the $\mathrm{P}$ and $\mathrm{P}^{2} / \mathrm{A}$ measures. A change in just one element affects these measures to such an extent that they become meaningless. As long as every cell in a matrix is potentially available for use as pattern element, increasing the fineness of grain will not help. As a matter of fact, two patterns constructed in a 20 by 20 matrix, for instance, with one-half of the cells lighted in a random fashion will be indiscriminable even when one is made the negative of the other, i.e., the $\mathrm{UE}$ value is maximized. Moments of area of such matrices would be practically identical and predict no discrimination. Such two patterns, however, may be quite discriminable under certain conditions even if their UE value is only 1 , but this type of pattern will of necessity begin to look like a polygon, e.g., two solid squares with just a "hole", in the middle of one, or an indented corner. While the UE measure would not predict discrimination here, some appropriate measure, such as moments of the perimeter, would. What this amounts to, as Polidora points out quite correctly, is that form measures must fit the conditions of their application. This includes not only such considerations as whether single or multiple stimuli are used in a display but also the size of the grain that has been used to construct and measure the stimuli.

\section{References}

Atneave, F., \& Amoult, M. D. The quantitative study of shape and pattern perception. Psychol. Bull., 1956, 53, 452-471.

Hochberg, J. E., Gleitman, H., \& McBride, P. D. Visual threshold as a function of simplicity of form. Amer. Psychologist, 1948 , 3, 341-342. (Abstract)

Polidora, B.J. Stimulus correlates of visual pattern discrimination by monkeys: Multidimensional analyses. Percept. \& Psychophys., $1966,1,405-414$

Zusne, L. Moments of area and of the perimeter of visual form as predictors of discrimination performance. J. exp. Psychol. $1965,69,213-220$.

(Accepted for publication December 9, 1966.)

\section{Reply to Zusne $^{1}$ by V. J. Polidora}

Zusne (1967) has raised three principal points of criticism of my paper (Polidora, 1966), and I shall discuss them in turn. 2

(1) Only four of the 15 dimensions were systematically varied, and "Inless the se other (11) dimensions are correlated with the se four, they will vary randomly, and it will be impossible to determine their respective contributions." The 11 dimensions did indeed vary randomly in terms of how frequently individual discrimination problems represented values of each dimension. I feel, however, that this fact does not detract from the validity of the results of the multiple regression and discriminant function analyses. The former analysis showed that discriminative performance significantly covaried with certain of these 11 dimensions (simple productmoment correlation), but that when the intercorrelations of each dimension were evaluated by partial correlation techniques, only the Unique Elements dimension remained significantly related to performance. The discriminant function analyses corroborated this conclusion. This result was obtained despite the fact that the Unique Elements dimension also varied randomly (as acknowledged on p. 411). Random sampling of different numbers of problems at each level of a (hypothetical) truly "valid" dimension will not necessarily destroy the predictive power of the "valid" dimension provided the size of the sample is adequate, nor will such an experiment necessarily reveal insignificant relationships simply because of random sampling.

(2) "All measures were pitted against one, Unique Elements, which, by definition, reflected any and all possible differences between two patterns, hence correlated with every one of the measures used." Zusne is in error on two counts: In the first place the statistical analyses pitted every dimension against every other; the results revealed the predominant contribution of the Unique Elements dimension. Secondly, the intercorrelations between Unique Elements and the remaining 14 dimensions were invariably low for all dimensions, correlating poorly with performance (range -0.04 to +0.12 ) and somewhat higher $(+0.05$ to +0.28$)$ for dimensions which did correlate significantly with performance (i.e., the six dimensions Zusne referred to). The important point, however, is that the significant partial correlation between Unique Elements and performance proves that the significant simple correlations were due solely to their intercorrelations with Unique Elements. 